\title{
Exploring Artifact Signals in Atom Probe Mass Spectra
}

\author{
F. Meisenkothen ${ }^{1}$ and E. B. Steel ${ }^{1}$ \\ 1. National Institute of Standards and Technology, Gaithersburg, MD, U.S.A.
}

A variety of artifact signals are commonly observed in atom probe tomography mass spectra. Some artifacts are immediately apparent (e.g. the background spectrum and peak tails), while some are more difficult to discern (e.g. DC field evaporation, ion feedback, and multi-hit detection events). A deeper understanding of mass spectrum artifacts should advance understanding of instrument behavior; produce more accurate analyses; help mitigate the effects of the artifacts; and provide ways to recover signal information that would otherwise be lost.

Nine specimens were included in the study - five CAMECA silicon pre-sharps, two electro-polished aluminum wires, one electro-polished tungsten wire, and one isotopically pure ${ }^{28} \mathrm{Si}$ blanket film $(\sim 175 \mathrm{~nm}$ thick). A laser-pulsed CAMECA LEAP-4000XSi ${ }^{\circledR}$ instrument (flight path length $=90 \mathrm{~mm}, 355 \mathrm{~nm} \mathrm{UV}$ ) was used for all of the experiments. The mono-isotopic silicon specimen was prepared via conventional in-situ lift-out and ion milling techniques in a dual beam FIB-SEM instrument [1]. Replicate data sets were collected to support the findings. Mass spectra and 3-D reconstructions were generated with the CAMECA Integrated Visualization and Analysis Software (IVAS ${ }^{\circledR}$ ). The ion information from each dataset was exported to binary *.ePOS files to permit further analyses, via custom software scripts (e.g. ion correlation histograms and multiplicity-specific mass spectra).

Three artifact signals commonly found in atom probe mass spectra were explored - DC field evaporation, thermal peak tails, and ion feedback. The study showed that even under acquisition conditions chosen to yield low background signals, DC field evaporation can make a significant contribution to the background spectrum. While the identities of these DC evaporated ion species were often unknown, the ions do retain position information. Spatial distribution maps indicated the background signal can be closely correlated with the crystallographic lattice-repeat that was reconstructed from the ions contained in the ranged ion peaks. Ion correlation histograms were used to explore the relationships between the constituent ions of multi-hit detection events. The ion correlation histograms also provided a means to detect some DC field evaporation events (Figure 1) [2,3]. The diagonal tracks, parallel to the red, $y=x$, reference line arise when two ions having different $\mathrm{m} / \mathrm{n}$ ratios field evaporate concomitantly, but delayed in time relative to the pulse. The further a diagonal track lies from the $\mathrm{y}=\mathrm{x}$ reference line, the larger is the time-of-flight (TOF) difference between the constituent ions in the pairs. A TOF difference spectrum, i.e. a histogram comprised of the absolute differences of the time-of-flight values between the first-of-multiples (FoM) and the second-of-multiples (SoM) in each ion pair (Figure 2), was developed to provide a means to identify and recover some of the DC field evaporated ions that would otherwise be lost to the background spectrum. Two-dimensional (2D) ion density contour plots were also used to provide spatially-resolved, chronological visualization of the ion emission process as a thermal pulse propagates through a silicon specimen tip. The results were consistent with the thermal mechanism that has been traditionally proposed to explain the peak tails in laser-pulsed mass spectra. Lastly, ion feedback was shown to be consistent with an artifact peak that is commonly observed in the mass spectrum of silicon [4]. 


\section{References:}

[1] K. Thompson, et al. Ultramicroscopy 107 (2007), p. 131.

[2] D.W. Saxey. Ultramicroscopy 111 (2011), p. 473.

[3] F. Meisenkothen, et al. Ultramicroscopy 159 (2015), p. 101.

[4] The authors would like to thank Drs. Ed Oltman, Robert Ulfig, Hugues Francois Saint Cyr, and Ty Prosa of CAMECA, Inc.; Drs. Ann Chiaramonti Debay and Norman Sanford of NIST-Boulder; and Dr. Karen Henry of Intel for their helpful discussions.

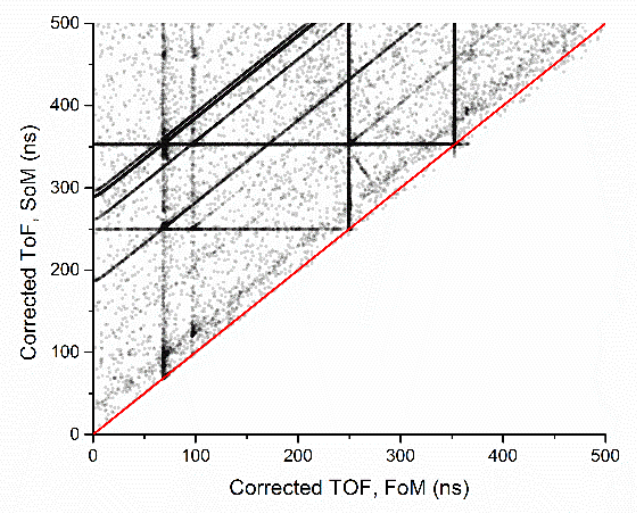

Figure 1. Corrected TOF ion correlation histogram for an aluminum specimen $\left[{ }^{27} \mathrm{Al}^{+}(353 \mathrm{~ns}),{ }^{27} \mathrm{Al}^{2+}\right.$ (249 ns), ${ }^{1} \mathrm{H}^{+}(68 \mathrm{~ns})$, and $\left.{ }^{1} \mathrm{H}_{2}{ }^{+}(96 \mathrm{~ns})\right]$. The ions in the diagonal tracks can be recovered from the background spectrum via a TOF difference spectrum.

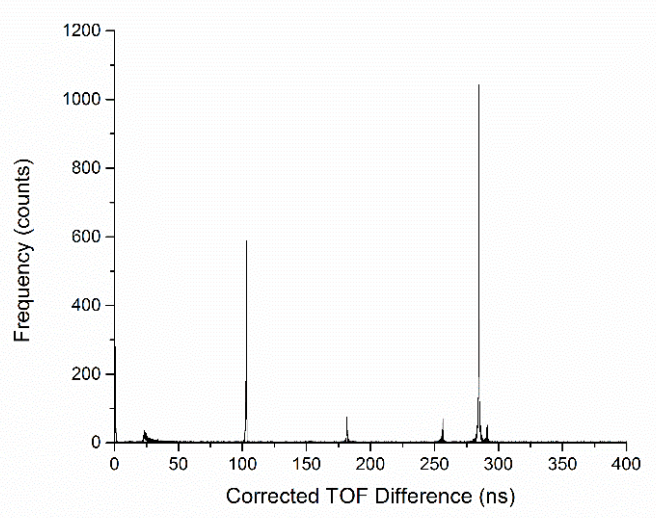

Figure 2. The TOF difference can be used in conjunction with the ion correlation histogram to determine the identity of constituent ions in multiplicity- 2 detection events. Detected ion pairs are: $0 \mathrm{~ns}$ (ion pairs on the $\mathrm{y}=\mathrm{x}$ reference line); $28 \mathrm{~ns}\left({ }^{1} \mathrm{H}^{+} /{ }^{1} \mathrm{H}_{2}{ }^{+}\right) ; 103 \mathrm{~ns}\left({ }^{27} \mathrm{Al}^{2+} /{ }^{27} \mathrm{Al}^{+}\right) ; 153 \mathrm{~ns}\left({ }^{1} \mathrm{H}_{2}{ }^{+} / 27 \mathrm{Al}^{2+}\right) ; 182$ ns $\left({ }^{1} \mathrm{H}^{+} / 27 \mathrm{Al}^{2+}\right) ; 257 \mathrm{~ns}\left({ }^{1} \mathrm{H}_{2}{ }^{+} / 27 \mathrm{Al}^{+}\right) ; 285 \mathrm{~ns}\left({ }^{1} \mathrm{H}^{+} / 27 \mathrm{Al}^{+}\right)$; and $291 \mathrm{~ns}\left({ }^{1} \mathrm{H}^{+} / 27 \mathrm{Al}^{1} \mathrm{H}^{+}\right)$. 